\title{
DEPRESSÃO E COMPORTAMENTO SUICIDA EM PACIENTES ONCOLÓGICOS HOSPITALIZADOS: PREVALÊNCIA E FATORES ASSOCIADOS
}

Priscila Caroline Fanger ${ }^{1}$, Renata Cruz Soares de Azevedo², Marisa lúcia Fabrício Mauro ${ }^{3}$, Daniela Dantas Lima4 ${ }^{4}$, Karla Cristina Gaspar ${ }^{5}$, Viviane Franco da Silva ${ }^{6}$, Wagner Tadeu Jurevicius do Nascimento ${ }^{7}$, Neury José Botega ${ }^{8 *}$

Trabalho realizado na Universidade Estadual de Campinas, Faculdade de Ciências Médicas, Departamento de Psicologia Médica e Psiquiatria, Campinas, SP

\section{*Correspondência:}

Faculdade de Ciências

Médicas, Universidade

Estadual de Campinas

Depto de Psiquiatria

Caixa Postal 6111

Campinas - SP

CEP: 13081-970

\section{RESUMO}

ОвJEtivo. Determinar as prevalências de depressão e de comportamento suicida em indivíduos com câncer internados em um hospital geral universitário e fatores associados a essas condições.

Métodos. O rastreamento de 5357 pacientes consecutivamente internados utilizou a Escala Hospitalar de Ansiedade e Depressão (HAD) e a seção comportamento suicida do Mini International Neuropsychiatric Interview (MINI). Análises univariadas, com ajustes para sexo e faixa etária, foram feitas tomando-se depressão e comportamento suicida como variáveis dependentes.

Resultados. Nos 675 pacientes com câncer, a prevalência de depressão foi 18,3\% (IC95\% $=15,4$ - 21,4). O risco para suicídio estimado pela respectiva seção do MINI foi de 4,7\% (IC95\% = 3,2 $6,7)$. A prevalência de depressão foi maior entre pacientes com câncer do que nos demais pacientes internados $(13,2 \% ; p=0,0009)$. Associaram-se à depressão $(p<0,05)$ : sexo feminino, menor escolaridade, maior tempo da doença, dor, uso de psicofármaco e risco de suicídio. Associaram-se ao risco de suicídio: dor e depressão.

Conclusão. As prevalências de depressão e de risco de suicídio em pacientes com câncer aconselham o uso de instrumentos simples de rastreamento e a inclusão, na anamnese, de perguntas mais específicas sobre essas condições clínicas.

UnITERmos: Transtorno depressivo. Comportamento autodestrutivo. Pacientes internados. Estatísticas hospitalares. Neoplasias.

\section{INTRODUÇÃO}

O impacto do câncer sobre a saúde mental tem sido estudado por diversos autores. Segundo metanálise publicada em 1997, as taxas de prevalência de transtorno mental em indivíduos com câncer oscilam entre $5 \%$ e $50 \%$ para distúrbios psiquiátricos em geral $^{1}$. Tal variação deve-se a diferenças clínicas (sítios ou estágios dos tumores), a ambientes diversos e a definições metodológicas distintas, como instrumentos de pesquisa utilizados e critérios de definição de transtorno mental ${ }^{2,3}$. Vários estudos indicam que a prevalência de distúrbios psiquiátricos graves em pacientes oncológicos é semelhante à da população geral ${ }^{1-4}$. Todavia, na metanálise supracitada foram encontrados níveis de depressão superiores ${ }^{1}$.
Em pesquisa com amostra populacional de indivíduos com câncer foi encontrada prevalência de 15,5\% de depressão maior de $15,5 \%{ }^{5}$. Em um recente estudo em que foram avaliados pacientes oncológicos ambulatoriais, de diferentes estágios e sítios da doença, foi encontrada uma taxa de 18,3\% de sintomas depressivos ${ }^{6}$. Entre os fatores associados à depressão nesses pacientes encontram-se estágio avançado da doença, intensidade da dor, viver sozinho, além da história pregressa de tratamento para depressão ${ }^{3-11}$

Alguns médicos podem ter dificuldade para detectar depressão em doentes oncológicos ${ }^{7}$. Uma possível explicação para isso é a falta de familiaridade com os sintomas de depressão, particularmente porque alguns sintomas somáticos, frequentemente associados à depressão, podem resultar do processo

1. Estudante de Medicina pela Faculdade de Ciências Médicas da Universidade Estadual de Campinas - FCM Unicamp, Campinas, SP

2. Doutorado em Saúde Mental - Professora Assistente - Doutor do Departamento de Psicologia Médica e Psiquiatria pela Faculdade de Ciências Médicas da Universidade Estadual de Campinas - FCM Unicamp, Campinas, SP

3. Doutorado em Saúde Mental - Psicóloga pesquisadora pela Universidade Estadual de Campinas - Unicamp, Campinas, SP

4. Graduação em Psicologia - Mestranda em Saúde Mental pela Universidade Estadual de Campinas - Unicamp, Campinas, SP

5. Graduação em Psicologia - Mestranda em Saúde Mental pela Universidade Estadual de Campinas - Unicamp, Campinas, SP

6. Mestrado e Doutoranda em Saúde Mental pela Universidade Estadual de Campinas - Unicamp, Campinas, SP

7. Graduação em Medicina - Médico pesquisador pela Universidade Estadual de Campinas, Campinas, SP

8. Médico, especialista em psiquiatria - Professor titular de Psicologia Médica da Faculdade de Ciências Médicas da Universidade Estadual de Campinas - FCM UNICAMP, Campinas, SP 
maligno subjacente. Além disso, os clínicos podem subestimar sintomas depressivos ao considerarem, equivocadamente, todos os pacientes com câncer "compreensivelmente deprimidos" 8 .

Outro dado clinicamente relevante é que o risco relativo de suicídio entre os pacientes com câncer, em relação à população geral, varia de 1,3 a 2,3 5, 12-15. Em um estudo de registro populacional de mortalidade foi encontrado significante risco de suicídio em homens (SMR 1.45; 95\% Cl 1.20-1.73). Em mulheres não houve significância estatística. Em ambos os sexos houve diminuição do risco relativo de suicídio com o aumento do tempo de diagnóstico ${ }^{16}$. Alguns fatores têm sido associados ao comportamento suicida em pacientes com câncer, tais como: sexo masculino, presença de depressão maior, doença maligna com pior prognóstico, declínio da função física, dor, delírio, fadiga, exaustão, falta de esperança, sensação de desamparo e deficiente apoio social 8,17-19.

Devido à escassez de estudos sobre transtornos psiquiátricos e risco de suicídio em pacientes com câncer na população brasileira, consideramos necessária a realização de trabalhos para avaliar tais comorbidades. Os objetivos deste estudo foram determinar a prevalência de depressão e de risco de suicídio em pacientes com câncer internados em um hospital geral universitário, bem como fatores associados a essas duas condições.

\section{MÉtodos}

\section{Delineamento}

Trata-se de um estudo transversal proveniente de dados da fase de rastreamento de um conjunto de estudos controlados e randomizados (Estudos de Intervenção Breve Oportuna - EIBO) que avaliaram, entre pacientes internados em um hospital universitário, a eficácia de uma intervenção (entrevista motivacional e contatos telefônicos periódicos, após a alta, ao longo de 12 meses) em casos de depressão, de tabagismo e de abuso ou dependência de álcool.

Este estudo desenvolveu-se no Hospital de Clínicas da Universidade Estadual de Campinas (HC-Unicamp), um hospital geral universitáro terciário com 380 leitos, localizado em Campinas (SP). O HC-Unicamp é um hospital terciário de referência para a rede pública de uma região metropolitana onde vivem cinco milhões de pessoas, atendendo casos de maior complexidade.

\section{Sujeitos}

Durante o período de recrutamento (outubro de 2007 a outubro de 2008), 6.276 indivíduos com idade igual ou superior a 18 anos foram internados no hospital (excetuando-se a Unidade de Terapia Intensiva e a Enfermaria de Psiquiatria). Desses, foi possível abordar 5.357 (85,3\%), dos quais 826 $(15,4 \%)$ tinham câncer. Tais valores, no caso de mais de uma internação do mesmo indivíduo no período, consideram apenas a primeira internação. Doze pacientes que sofriam de câncer recusaram-se a participar, e 138 (16,7\%) foram excluídos. Os motivos de exclusão foram: condições clínicas impossibilitando a entrevista, tais como, mau estado geral, distúrbio do nível de consciência e déficit cognitivo (51\%), alta hospitalar prevista dentro de poucas horas (23\%), residência fora do Estado de São Paulo $(21 \%)$ e inexistência de telefone para contatos durante o seguimento (5\%). Assim, 675 pacientes com câncer foram incluídos nesse estudo.

\section{Instrumentos}

O questionário utilizado continha dados sociodemográficos e informações sobre as condições de saúde atual e a razão da internação hospitalar, coletados do prontuário médico, bem como escalas psicométricas:

Escala Hospitalar de Ansiedade e Depressão (HAD) ${ }^{20}$, com sete itens de múltipla escolha para avaliação de ansiedade e sete para depressão, foi desenvolvida para ser aplicada em pacientes não-psiquiátricos com morbidades clínicas. A determinação dos "casos" de depressão foi estabelecida por pontuação igual ou maior que oito na sub escala de depressão. Tal ponto de corte corresponde ao previamente adotado em estudo validação do instrumento realizado em nosso meio ${ }^{21}$.

Seção de risco de suicídio do Mini International Neuropsychiatric Interview (MINI-plus, versão 5.0 plus) ${ }^{22,23}$, a qual avalia o risco de suicídio por meio de cinco perguntas, abarcando o período dos últimos 30 dias: Pensou que seria melhor estar morto, ou desejou estar morto? (1 ponto); Quis fazer algum mal a si mesmo? (2 pontos); Pensou em suicídio? (6 pontos); Pensou numa maneira de se suicidar? (10 pontos); Tentou o suicídio? (10 pontos). Uma pergunta final questiona sobre tentativa de suicídio ao longo da vida (4 pontos). O risco de suicídio é definido segundo a pontuação final: "baixo" (pontuação 1 a 5), "moderado" (pontuação 6 a 9) e "alto" (pontuação $\geq 10$ ). Apenas pacientes com escore indicativo de risco moderado/grave foram considerados "com risco de suicídio" na análise estatística.

Alcohol Use Disorder Identification Test - AUDIT, compreende 10 questões de múltipla escolha e identifica risco de abuso de álcool/dependência quando a pontuação (que varia de 0 a 40) é igual ou maior que $8^{24,25}$.

O tabagismo foi abordado por meio de uma única questão: "Você fumou diariamente durante o último mês?". A intensidade da dor foi avaliada por meio de valores indicados pelo paciente, em uma escala de pontuação de 0 a 10 , correspondentes às intensidades no dia da realização da entrevista e nos sete dias precedentes. Para efeito de análise, considerou-se qualquer valor diferente de zero como "presença" de dor. Os pacientes tiveram as doenças classificadas segundo o aparelho acometido, conforme os capítulos da CID-10 (Classificação Internacional das Doenças).

A entrevista de rastreamento era realizada nos dias úteis, à beira do leito, entre 24 a 72 horas após a internação. Dezessete estudantes de medicina foram treinados para administrá-la. 0 treinamento consistiu de três reuniões de duas horas de duração e compreendeu: introdução ao protocolo da pesquisa, simulações de entrevistas, e duas entrevistas com pacientes sob supervisão de médicos psiquiatras.

\section{Análise de dados}

Prevalências de depressão e de risco de suicídio (este último, segundo definido pelo $\mathrm{MINI}$ ), com respectivos intervalos de confiança de 95\% (IC 95\%), foram calculadas. Essas duas condições foram tomadas como variáveis dependentes em testes do Qui quadrado. Como variáveis independentes, incluíram-se informações sociodemográficas e clínicas, essas últimas provindas dos registros de prontuário ou de respostas a perguntas sumárias constantes do formulário de rastreamento. Quando pertinente, os 
resultados mostrados em tabela foram ajustados, por sexo e por faixa etária, por meio de regressão logística múltipla.

Este estudo foi aprovado pelo Comitê de Ética em Pesquisa da instituicão. Os pacientes assinaram termo de consentimento livre e esclarecido. Os pesquisadores informavam aos médicos assistentes sobre casos com transtorno mental grave ou risco de suicídio. O programa estatístico utilizado foi o SAS, versão 8.02.

\section{Resultados}

A média de idade dos pacientes incluídos no estudo foi inferior à dos excluídos (56,4 vs 60,1; $p=0,04$; Mann-Whitney). Não foram encontradas diferenças estatísticas quanto a sexo e uso de psicofármacos. A Tabela 1 resume as principais características dos pacientes com câncer.

Entre os pacientes incluídos, encontrou-se a seguinte distribuição, segundo o sítio do tumor: aparelho digestivo (40,4\%), hematológico $(15,8 \%)$, genitourinário $(15,4 \%)$, respiratório $(9,9 \%)$, pele $(7,6 \%)$, sistema nervoso $(6,0 \%)$, endócrino $(3,2 \%)$, osteomuscular $(1,5 \%)$ e outros $(3,1 \%)$.

A prevalência global de depressão foi de 18,3\% (IC95\% = $15,4$ - 21,4). A prevalência foi de 23\% (IC95\% = $18-28,7)$ para as mulheres e de 15,4\% (IC95\% = 12,1 - 19,2) para os homens. Ao se compararem pacientes com câncer com todos os que não tinham tal patologia, encontraram-se níveis de depressão superiores nos primeiros (18,3\% e 13,2\%, respectivamente; $p=0,0009$, ajustado por sexo e faixa etária). As características clínicas e demográficas dos pacientes oncológicos com depressão encontram-se na Tabela 2.

A prevalência de risco de suicídio foi de 4,8\% (IC95\% = 3,3 - 6,7) e não foi estatisticamente diferente da dos outros pacientes $(5,2 \% ; p=0,6)$. A prevalência de risco de suicídio não variou entre sexos, faixas etárias, estados civis, escolaridade ou religiosidade. Foi maior entre os pacientes que sentiram dor na última semana, comparados aos que não sentiram $(6,8 \%$ e $1,9 \%$, respectivamente; $p=0,006)$, como também entre os que se encontravam deprimidos $(15,4 \%$ e $2,4 \% ; p=0,0001)$, segundo regressão logística múltipla, ajustada por sexo e faixa etárias (resultados não mostrados em tabela).

\section{Discussão}

Este estudo foi capaz de abordar e avaliar, por meio de instrumentos padronizados, um grande número de pacientes com câncer, internados em um hospital universitário. Em termos aproximados, mostrou que um, em cada cinco, sofre de depressão e que $5 \%$ têm risco de suicídio, sendo este maior entre os que têm dor e os que se encontram deprimidos. Consideramos como maior mérito deste estudo o elevado número de pacientes avaliados em um centro universitário de alta complexidade, por meio de um esquema de rastreamento que se manteve ao longo de 13 meses.

Há, no entanto, limitações metodológicas que devem ser levadas em conta. Em primeiro lugar, há falta de algumas informações, tais como diferentes tipos de cânceres, estadiamento e gravidade de eventuais complicações. Esses dados costumam ser incluídos, em vários estudos na área de oncologia, entre as variáveis independentes ${ }^{3,5,6}$. 0 número limitado de informações deveu-se ao fato de o rastreamento de mais de 5000 indivíduos

\begin{tabular}{|c|c|}
\hline Características & $\mathrm{N}(\%)$ \\
\hline \multicolumn{2}{|l|}{ Sexo } \\
\hline Masculino & $423(62.7)$ \\
\hline \multicolumn{2}{|l|}{ Idade (anos) } \\
\hline Média (desvio padrão) & $56,4(15,4)$ \\
\hline Mediana & 57 \\
\hline \multicolumn{2}{|l|}{ Faixa etária } \\
\hline$<=47$ & $169(25,0)$ \\
\hline $48-57$ & $173(25,6)$ \\
\hline $58-67$ & $171(25,3)$ \\
\hline$>=68$ & $162(24,0)$ \\
\hline \multicolumn{2}{|l|}{ Estado civil } \\
\hline Casado/Amasiado & $449(66,8)$ \\
\hline Solteiro & $85(12,7)$ \\
\hline Divorciado/Separado & $69(10,3)$ \\
\hline Viúvo & $69(10,3)$ \\
\hline \multicolumn{2}{|l|}{ Atividade ocupacional } \\
\hline Dona de casa & $67(10,1)$ \\
\hline Sem atividade & $412(62,3)$ \\
\hline Em atividade & $182(27,5)$ \\
\hline \multicolumn{2}{|l|}{ Anos de escolaridade } \\
\hline Média (desvio padrão) & $\begin{array}{cc}5,6 & (4,2) \\
388 & (578)\end{array}$ \\
\hline 0 a 4 anos & $388(57,8)$ \\
\hline & \\
\hline $\begin{array}{l}\text { Declaram ter religiao } \\
\text { Não }\end{array}$ & $36(5,4)$ \\
\hline $\operatorname{Sim}$ & $636(94,6)$ \\
\hline \multicolumn{2}{|l|}{ Frequência a cultos religiosos no último mês } \\
\hline Nenhuma & $302(48,1)$ \\
\hline 1 ou mais & $326(51,9)$ \\
\hline \multicolumn{2}{|l|}{ Duração do câncer (meses) } \\
\hline Média (desvio padrão) & $22,6(34,6)$ \\
\hline Mediana & 9,0 \\
\hline$<10$ & $176(51,2)$ \\
\hline 10 ou mais & $168(48,8)$ \\
\hline \multicolumn{2}{|l|}{ Dor } \\
\hline Não & $270(40,4)$ \\
\hline Sim & $398(59,6)$ \\
\hline \multicolumn{2}{|l|}{ No dia da entrevista } \\
\hline Não & $411(61,6)$ \\
\hline $\operatorname{Sim}$ & $256(38,4)$ \\
\hline \multicolumn{2}{|l|}{ Toma/já tomou algum psicofármaco } \\
\hline Não & $507(75,8)$ \\
\hline $\operatorname{Sim}$ & $162(24,2)$ \\
\hline Frequência de tabagismo & $95(14,1)$ \\
\hline Frequência de dependência/uso abusivo de álcool & $54(8,0)$ \\
\hline \multicolumn{2}{|l|}{ Frequência de risco de suicídio } \\
\hline Sem Risco & $639(95,2)$ \\
\hline Com risco & $32(4,8)$ \\
\hline
\end{tabular}




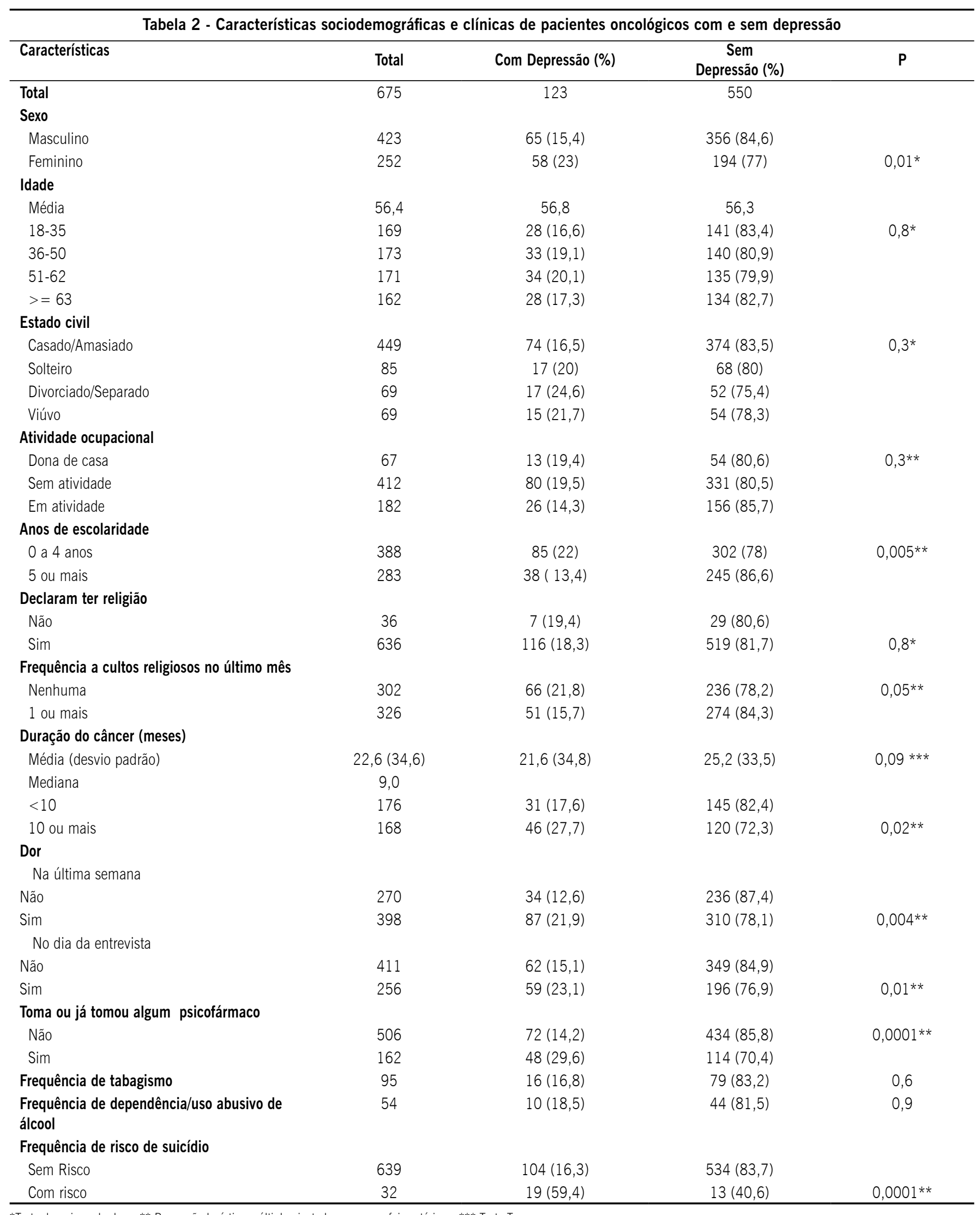

*Teste do qui quadrado ** Regressão logística múltipla ajustada por sexo e faixa etária $\quad$ *** Teste T 
exigir instrumentos válidos e concisos, que não cansassem o paciente, nem demandassem tempo extra do entrevistador na pesquisa de prontuários. Este estudo não foi idealizado para se aprofundar, especificamente, em aspectos clínicos de pacientes com câncer. A avaliação de dor, por exemplo, foi sumária, e a dicotomização que fizemos na análise estatística impediu que considerássemos a frequência de depressão em diferentes graus de dor. Fizemos tão somente o registro do sítio do câncer, mas não incluímos essa variável nas análises estatísticas. Essas, por sua vez, não avançaram até um modelo de regressão logística, justamente pelo caráter descritivo do estudo (não houve a priori hipóteses a serem testadas) e pela limitação que tivemos na coleta de informações clínicas mais aprofundadas, direcionadas especificamente para pacientes oncológicos, que pudessem compor uma relação confiável de variáveis independentes.

Em segundo lugar, a prevalência de depressão foi obtida por meio do instrumento de rastreamento mais utilizado em estudos europeus em pacientes com câncer ${ }^{3}$. A HAD evita itens que contenham sintomas somáticos, com o intuito de diminuir a ocorrência de casos falso-positivos ${ }^{20}$. 0 ponto de corte aqui definido para a definição de "caso de depressão" obedeceu a critérios mais estritos, estabelecidos em estudo prévio de validação, realizado na mesma instituição ${ }^{21}$. Ainda assim, apesar desses cuidados, não se realizou entrevista psiquiátrica padronizada para confirmar depressão.

Em terceiro lugar, é importante lembrar que, embora ideação suicida e tentativa prévia de suicídio sejam importantes preditores de suicídio, não podemos afirmar que esses dois fatores bastam para uma avaliação de "risco" de suicídio (como denominado pelo $\mathrm{MINI}$ ) entre pacientes oncológicos internados no hospital geral. Nesse ambiente, sabe-se que há pelo menos três grupos de pacientes com risco de suicídio: os que foram internados devido a uma tentativa de suicídio; os pacientes sob o estresse de uma doença crônica ou, então, de um diagnóstico recentemente revelado; e os pacientes em estado confusional (delirium) acompanhado de agitação e impulsividade ${ }^{26}$. Esse último grupo foi excluído do estudo, pois não estaria em condições de, adequadamente, responder o questionário de rastreamento. Feita essa ressalva, é importante reconhecer que a seção de "risco de suicídio" do MINI permite avaliar um aspecto geralmente desconsiderado nas avaliações clínicas, que vai desde desejos passivos de morte até o planejamento e consecução de uma tentativa de suicídio.

A prevalência de depressão entre pessoas acometidas por câncer varia substancialmente entre os diferentes estudos, devido ao emprego de distintas estratégias metodológicas, características de serviços e critérios operacionais empregados 1-3. Ainda assim, a prevalência que encontramos foi superior aos $5 \%$ de alguns estudos que utilizaram critérios diagnósticos estritos, como por exemplo, episódio depressivo grave (major depression) ${ }^{8,9}$. É inferior, no entanto, a um trabalho recente no qual também se utilizou a HAD em 94 pacientes com câncer de esôfago, chegando-se a uma cifra de $42 \%$ para casos "prováveis e possíveis de depressão" ${ }^{27}$. Entretanto é difícil realizarmos uma comparação já que grande parte dos trabalhos avalia grupos específicos de pacientes oncológicos.

Segundo a meta-análise de Spijker et al. ${ }^{1}$ os níveis de depressão entre os pacientes com câncer são maiores que os encontrados na população geral. No entanto, faltam estudos que comparem estes indivíduos com pacientes acometidos por outras patologias. No presente estudo, a prevalência de depressão entre os pacientes oncológicos, tomados em conjunto, foi maior, quando comparada à dos demais pacientes internados. Entre as possíveis explicações, há o fato de a maioria das internações ser de casos graves, o que acarreta grande impacto negativo nas atividades de vida diária, no campo das relações pessoais, no grau de bem-estar físico e nos sentimentos de prazer e de esperança, esses últimos presentes em vários itens da sub escala de depressão da HAD.

A maioria dos fatores associados à depressão foi concordante com outros estudos, tais como sexo feminino, maior tempo de doença, presença de dor, uso de psicofármaco, risco de suicídio, menor escolaridade, menor frequência a cultos religiosos e presença de dor ${ }^{2,3,6-10}$.

A taxa de $4,7 \%$ de risco de suicídio entre os pacientes com câncer coincide com dados de estudo anterior, feito no mesmo hospital, que utilizou o MINI em amostra aleatória de 253 indivíduos internados ${ }^{28}$. Quanto ao risco de suicídio, alguns fatores aqui encontrados, como intensidade da dor, uso de psicofármacos ao longo da vida e depressão, são compatíveis com achados de outros trabalhos ${ }^{5,8,17,18,27}$. Não foram encontrados níveis superiores de risco de suicídio no sexo masculino e entre indivíduos divorciados/separados, diferentemente de outros estudos ${ }^{15-17}$.

Este estudo apontou maior prevalência de depressão em pacientes com câncer do que em pacientes acometidos por outras doenças. Isso reforça a importância da detecção e do tratamento dessa condição, haja vista que a depressão afeta a adesão ao tratamento, bem como influencia a evolução do câncer e a qualidade de vida. Instrumentos simples, como a HAD, ou algumas perguntas mais específicas sobre depressão, feitas pelo profissional de saúde, devem ser rotineiramente incluídas na avaliação de pacientes acometidos por câncer. Tal medida visa otimizar a detecção e a intervenção nos casos de depressão, ampliando a qualidade da assistência prestada.

\section{AGRADECIMENTO}

1 - Este estudo foi feito com o apoio financeiro da Fundação de Amparo à Pesquisa do Estado de São Paulo, processos 06/61885-6 e 07/59343-3. Agradecemos Cleide Aparecida Moreira Silva, pela assessoria estatística.

\section{SUPORTE FINANCEIRO:}

Fundação de Amparo à Pesquisa do Estado de São Paulo - FAPESP

\section{Conflito de interesse: não há}

\section{SUMMARY}

DEPRESSION AND SUICIDAL BEHAVIOR OF CANCER INPATIENTS: PREVALENCE AND ASSOCIATED FACTORS

OBJECTIVE. To determine prevalence rates of depression and suicidal behavior among cancer inpatients and factors associated with these conditions. Methods. A total of 5357 patients consecutively admitted to a university hospital were assessed by means of the Hospital Anxiety and Depression Scale (HAD) and the suicide risk section of the Mini International Neuropsychiatric Interview (MINI). Univariate analyses adjusted for gender and 
FANGER PC ET AL.

age groups were performed with depression and suicide risk as dependent variables.

RESULTS. Of those assessed, 675 had cancer. The prevalence rates for depression and suicide risk were $18.3 \%(95 \% \mathrm{Cl}=15.4$ - 21.4) and 4.7\% (95\% Cl = 3.2 - 6.7) respectively. Depression was more frequently found in cancer bearers than in the other inpatients (13.2\%; $p=0.0009$ ). Female gender, low schooling level, long time of disease, pain, use of psychotropic drugs and suicide risk were associated with depression ( $p<0.05$ ). Pain and depression were associated with suicide risk.

Conclusion. Because prevalence rates of depression and suicide risk are high among cancer inpatients, simple screening instruments and specific questions during interviews are needed to detect these clinical conditions. [Rev Assoc Med Bras 2010; 56(2): 173-8]

KEY WORDS: Depressive disorder. Self-injurious behavior. Inpatients. Hospital statistics. Neoplasms.

\section{REFERÊNCIAS}

1. Van't Spijker A, Trusburg RW, Duivenvoorden HJ. Psychological sequelae of cancer diagnosis: a meta-analytical review of 58 studies after 1980. Psychosom Med. 1997;59:280-93.

2. Härter M, Reuter K, Aschenbrenner A, Schretzmann B, Marschner N, Hasenburg $A$, et al. Psychiatric disorders and associated factors in cancer: results of an interview study with patients in inpatient, rehabilitation and outpatient treatment. Eur J Cancer. 2001;37:1385-93.

3. Wasteson E, Brenne E, Higginson IJ, Hotopf M, Lloyd-Williams M, Kaasa S, et al. Depression assessment and classification in palliative cancer patients: a systematic literature review. Palliat Med. 2009 (in press)

4. Kadan-Lottick NS, Vanderwerker LC, Block SD, Zhang B, Prigerson HG. Psychiatric disorders and mental health service use in patients with advanced cancer. Cancer. 2005;104:2872-81.

5. Rasic DT, Belik SL, Bolton JM, Chochinov HM, Sareen J. Cancer, mental disorders, suicidal ideation and attempts in a large community sample. Psychooncology. 2008;17:660-7.

6. Brintzenhofe-Szoc KM, Levin TT, Yuelin Li BS, Kissane DW, Zabora JR. Mixed Anxiety/Depression Symptoms in a Large Cancer Cohort: prevalence by cancer type. Psychosomatics. 2009; 50:383-91.

7. Kugaya A, Akechi T, Okuyama T, Nakano T, Mikami I, Okamura H, et al. Prevalence, predictive factors, and screening for psychologic distress in patients with newly diagnosed head and neck cancer. Cancer. 2000;88:2817-23.

8. ChochinovHM. Depression incancerpatients. LancetOncol. 2001;2:499-505.

9. Akechi T, Nakano T, Okamura H, Ueda S, Akizuki N, Nakanishi T, et al. Psychiatric disorders in cancer patients: descriptive analysis of 1721 psychiatric referrals at two Japanese Cancer Center Hospital. Jpn J Clin Oncol. 2001;31:188-94.

10. Akechi T, Okamura H, Nishiwaki Y, Uchitomi Y. Psychiatric disorders and associated and predictive factors in patients with unresectable nonsmall cell lung carcinoma: a longitudinal study. Cancer. 2001;92:2609-22.
11. Jenkins C, Carmody TJ, Rush AJ. Depression in radiation oncology patients: a preliminary evaluation. J Affect Disord.1998;50:17-21.

12. Louhivuori KA, Hakama M. Risk of suicide among cancer patients. Am J Epidemiol.1979;109:59-65.

13. Fox BH, Stanek III EJ, Boyd SC, Flannery JT. Suicide rates among cancer patients in Connecticut. J Chronic Dis.1982;35:89-100.

14. Allebeck P, Bolund C, Ringbäck G. Increased suicide rate in cancer patients: a Cohort study based on the Swedish Cancer-Environment Register. J Clin Epidemiol 1989;42:611-6.

15. Hem E, Loge JH, Haldorsen T, Ekeberg O. Suicide Risk in Cancer Patients from 1960 to 1999. J Clin Oncol .2004;22:4209-16.

16. Robinson D, Renshaw C, Okello C, Mollen H, Davies EA. Suicide in cancer patients in South East England from 1996 to 2005: a population-based study. Br J Cancer. 2009;(101)198-201.

17. Kendal WS. Suicide and cancer: a gender-comparative study. Ann Oncol. 2007; 18:381-7.

18. Akechi T, Nakano T, Akizuki N, Nakanishi T, Yoshikawa E, Okamura, H, et al. Clinical factors associated with suicidality in cancer patients. Jpn J Clin Oncol. 2002;32:506-11.

19. Akechi T, Okamura H, Nishiwaki Y, Uchitomi Y. Predictive factors for suicidal ideation in patients with unresectable lung carcinoma: a 6-month Follow-Up Study. Cancer. 2002;95:1085-93.

20. Zigmond AS, Snaith RP. The Hospital Anxiety and depression Scale. Acta Psychiatr Scand.1983;67:361-70.

21. Botega NJ, Bio MR, Zomignani MA, Garcia Jr C, Pereira WA. Transtornos de humor em enfermaria de clínica médica e validação de escala de medida (HAD) de ansiedade e depressão. Rev Saúde Publica.1995;29:355-63.

22. Sheehan DV, Lecrubier Y, Sheehan KH, Amorim P, Janavs J, Weiller E, et al. The Mini-International Neuropsychiatric Interview (M.I.N.I.): the development and validation of a structured diagnostic psychiatric interview for DSM-IV and ICD-10. J Clin Psychiatry. 1998;59(Suppl 20):22-33.

23. Amorim P. Mini International Neuropsychiatric Interview (MINI): validação de entrevista breve para diagnóstico de transtornos mentais. Rev Bras Psiquiatr. 2000;22:106-15.

24. Barbor TE, La Fuente JR, Saunders J, Grant M. AUDIT: The alcohol use disorders identification test: guidelines for use in primary care. WHO (PSA/92) 1992;4:1-29.

25. Figlie NB, Pillon SC, Laranjeira RR, Dunn J. AUDIT identifica a necessidade de interconsulta específica para dependentes de álcool no hospital geral? J Bras Psiquiatr. 1997;46: 589-93.

26. Tishler CL, Reiss NS. Inpatient suicide: preventing a common sentinel event. Gen Hosp Psychiatry. 2009;31:103-9.

27. Bergquist $H$, Ruth $M$, Hammerlid. Psychiatric morbidity among patients with cancer of the esophagus or the gastro-esophageal junction: a prospective, longitudinal evaluation. Dis Esophagus. 2007;20:523-9.

28. Ferreira $\mathrm{MH}$, Colombo ES, Guimarães PS, Soeiro RE, Dalgalarrondo P, Botega NJ. Suicide risk among in patients at a university general hospital. Rev Bras Psiquiatr. 2007;29:51-4

Artigo recebido: 23/05/09 Aceito para publicação: 17/01/10 